\title{
A Transaction Execution Engine Architecture for Multiplayer Online Games
}

\author{
Ian Lintault \\ Intel Corporation \\ 3-1-1 Marunouchi, Chiyoda-ku, Tokyo, Japan 100-0005 \\ ian.lintault@intel.com
}

\begin{abstract}
In the pursuit of massively multiplayer online games, efficient engines capable of processing sophisticated game scenarios are necessary to support large scale virtual worlds. By applying microprocessor microarchitectural design techniques to game server software design, functional parallelism inherent in complex game transactions can be exploited, augmenting server performance.

In a given gaming scenario, complex game transactions can be modeled as data flow graphs. An individual graph represents the collective atomic operations of a particular transaction. Analysis of data flow graphs provides a mechanism that identifies opportunities for parallelism by exposing data dependencies.

This poster presents an engine architecture that supports spatial and temporal parallel execution of complex game transactions expressed as data flow graphs. The engine takes advantage of data flow enhancement techniques such as dynamic out-of-order execution to enhance performance.
\end{abstract}

\section{Categories and Subject Descriptors}

D.2.11 [Software]: Software EngineeringSoftware Architectures; J.m [Computer Applications]: Miscellaneous; I.6.7 [Computing Methodologies]: Simulation and ModelingSimulation Support Systems

\section{General Terms}

Theory Design Performance

\section{Keywords}

Game architecture, Data flow architecture, Transaction level parallelism, Distributed simulation, Transaction execution, Dynamic scheduling, Task scheduling, Online Games

\section{SUMMARY}

The increasing complexity of large scale game scenarios demands an appropriate increase in game server performance. Previous work [1] on game server architectures identify parallelism as a potential method of increasing performance.

Aside from parallelism exposed by executing transactions simultaneously in separate threads common to typical thread

Copyright is held by the author/owner. SIGCOMM'04 Workshops, Aug. 30+Sept. 3, 2004, Portland, Oregon, USA. ACM 1-58113-942-X/04/0008. pool architectures [2], a previously untapped source of functional parallelism inherent in some transactions can also be exploited by analysis on a finer scale.

Consider a system model that has multiple clients sending requests to a game server system. The servers are responsible for executing transactions received from clients and distributing the simulation state. Transactions vary in complexity and may require further processing by servers in other tiers of the system architecture.

In this model we assume transactions are of two types, simple and complex. A simple game operation represents a single atomic operation. A complex transaction contains a collection of atomic operations that must be executed in a specified order due to data dependencies.

Complex transactions can be expressed as a type of acyclic data dependence graph (DDG) known as a data flow graph (DFG). The data flow graph nodes represent a single atomic game operation and the graph arcs represent a true data dependency. These graphs can be analyzed for fine-grained parallelism opportunities.

A transaction execution engine was developed that analyzes and exploits parallelism to increase the performance of online gaming systems. The engine features a dynamic core [3] which schedules out-of-order execution of atomic game operations. Functional units implemented as dynamically linked libraries (DLLs) execute atomic operations either locally or remotely in an asynchronous fashion. The processing pipeline stages and functional units are realized as object oriented software.

\section{REFERENCES}

[1] Abdelkhalek, A., Bilas, A., And Moshovos, A. Behavior and performance of interactive multi-player game servers. In Proc. International IEEE Symposium on the Performance Analysis of Systems and Software ISPASS-2001 (2001).

[2] Schmidt, D., And Vinoski, S. Comparing alternative programming techniques for multi-threaded servers. SIGS C++ Report 8, 2 (February 1996).

[3] Beckmann, C., and Polychronopoulos, C. Microarchitecture support for dynamic scheduling of acyclic task graphs. In Proceedings 25th Annual International Symposium on Microarchitecture (Portland, Oregon, 1992), IEEE Computer Society Press, pp. 140-148. 